\title{
The Influence of Temperature on Coumarin 153 Fluorescence Kinetics
}

\author{
Krzysztof Dobek
}

Received: 5 November 2010 / Accepted: 10 January 2011 / Published online: 19 January 2011

(C) The Author(s) 2011. This article is published with open access at Springerlink.com

\begin{abstract}
The influence of temperature varied in the range $183 \mathrm{~K}-323 \mathrm{~K}$ on the fluorescence quantum yield, fluorescence lifetime, absorption and emission transition moments and non-radiative deactivation rate was determined for the well known and largely used dye Coumarin 153, dissolved in 1chloropropane. The Kennard-Stepanov relation connecting the absorption and emission spectra was used to check for the presence of more than one absorbing/emitting species and to investigate whether intramolecular vibrational redistribution completes in the $\mathrm{C} 153$ excited $S_{1}$ state before the emission takes place. The emission spectrum corresponding to $S_{1} \rightarrow S_{0}$ transition, was fitted at each temperature to the model function including the information on the dye vibrational modes coupling. In this way the displacement in equilibrium distance for the most active vibrational mode was determined for $\mathrm{C} 153$ in $S_{1}$ and in $S_{0}$. Using the temperature dependence of the fluorescence decay time and quantum yield, the nonradiative deactivation rate was determined. Its temperature dependence was compared to that calculated using the theoretical model with the most active vibrational mode displacement values taken from steady-state spectra analysis. The somewhat surprising dependence of the fluorescence decay time and quantum yield on temperature was related to non-trivial coupling between low-frequency vibrational modes of $\mathrm{C} 153$ in the excited and ground states.
\end{abstract}

\section{K. Dobek $(\bowtie)$}

Faculty of Physics, Adam Mickiewicz University,

Umultowska 85,

61-614 Poznań, Poland

e-mail: dobas@amu.edu.pl

K. Dobek

Faculty of Physics, Astronomy and Informatics,

Nicolaus Copernicus University,

Toruń, Poland
Keywords Coumarin $153 \cdot 1$-chloropropane . Fluorescence $\cdot$ Kinetic $\cdot$ Temperature

\section{Introduction}

Coumarin 153 (C153, Scheme 1) is a well known dye, probably one of the most often used in fundamental studies of the environment influence on fluorescence properties of a molecule. It is a fairly simple molecule, strongly emissive, which can be used as a perfect model for much more complicated systems. Despite great interest in this molecule, as a representative of a large family of coumarin dyes, its properties, including the intramolecular deactivation, have not been fully recognized yet. This work has been undertaken to establish the temperature influence on $\mathrm{C} 153$ absorptive and emissive properties. The dye was dissolved in 1-chloropropane (ClP), a solvent of moderately polarity, widely assumed to interact exclusively non-specifically. Thus, results presented below can serve as a model for the same dye dissolved in other solvents, possibly also for other molecules, like some coumarin derivatives, and most of all it presents a methodology of studying selected temperature dependent effects present in many other dyes.

At room temperature, absorption of light from the $\mathrm{C} 153$ ground to the excited electronic state, $S_{0} \rightarrow S_{1}$, is of $\pi \pi^{*}$ type and connects two electronic states of the two conformers: I, with the julolidine group in syn conformation, and II with the same group in anti conformation $[1,2]$. In supersonic jet conditions the energy difference between the origins of $S_{0} \rightarrow S_{1}$ transition for both conformers is equal only $188 \mathrm{~cm}^{-1}$. In the ground state conformer I seems to be the dominating form, at least in the jet-cooled conditions [1]. The intensity of absorbed light is significant as the 


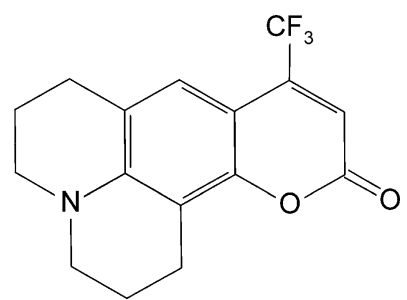

Scheme 1 Coumarin 153 (C153) structure

molecule has a relatively high molar absorption coefficient of $\sim 20,000 \mathrm{lmol}^{-1} \mathrm{~cm}^{-1}$ [2]. Excitation leads to a change in C153 dipole moment, from 6.6 D to $9.7 \mathrm{D}$, as derived from thermochromic studies (this parameter's value is still under debate) [3]. This property makes $\mathrm{C} 153$ a useful solvatochromic probe, especially for emission studies. In supersonic jet the fluorescence takes place mainly from the excited conformer II [2]. Fluorescence quantum yield of 0.1 in water increases to 0.9 in cyclohexane [4]. Even if in solution the fluorescence activity of both conformers cannot be excluded, their properties must be very similar, as the transition dipole moment for fluorescence has been found to be constant (in the range of experimental error), despite changes in polarity of the environment [5]. Indeed, this similarity has been confirmed by ab-initio calculations in Ref. [6]. The fluorescence decay time fluctuates at room temperature in the range of 4-6 ns in a large set of solvents without any correlation with their polarity [5]. Thus, an increase in the environment polarity, which through solvation leads to a drop in the $S_{1}-S_{0}$ energy gap, results in an increase in non-radiative deactivation, as can be deduced from the influence of environment polarity on quantum yield. These simple properties make C153 so popular, as in most cases the signal resulting from the molecule intramolecular deactivation can be easily separated from the one connected to intermolecular interactions, often quite complicated in nature. Because of a significant change in the dipole moment value, the molecule has been widely used as a solvatochromic $[7,8]$ or solvation dynamic probe [9-14]. It has been also suggested as an effective probe of its environment hydrogen-bondingability $[7,13,15]$, a thesis negated by some [16]. In response to its popularity, several attempts at theoretical calculations of the $\mathrm{C} 153$ geometry and energy have been undertaken [6, 17]. These works have confirmed the presence of both conformers in the $\mathrm{C} 153$ first excited state, reporting them to be almost equal in energy and charge distribution.

At the vibrational level, deactivation of $\mathrm{C} 153$ does not look as simply as drawn above. Almost three decades ago Ernsting et al. [18] reported C153 having a surprisingly dense vibronic spectrum of the $\pi \pi^{*}$ transition. Then, Topp et al. [1] have shown results indicating for the first time the presence of two C153 conformers and differences in their supersonic-jet fluorescence spectra. Later, Ernsting et al. [2] have shown in supersonic jet that an excitation energy by $\geq 200 \mathrm{~cm}^{-1}$ higher than the conformer I origin transition, lead to an effective intramolecular vibrational redistribution (IVR). The final state of IVR is the excited conformer II, and this is the most important fluorescing species, while a smaller excess in excitation energy leaves the probe in conformer I state. Additionally, the same authors reported that $\mathrm{C} 153$ fluorescence decay was most probably controlled by non-trivial vibrational coupling between $S_{1}$ and $S_{0}$ vibrational states. It was shown that energy exceeding by $+44 \mathrm{~cm}^{-1}$ and $+134 \mathrm{~cm}^{-1}$ the conformer I origin excitation energy can lead to an unexpected enhance in oscillator strength in the observed fluorescence of the second and third member of the $132 \mathrm{~cm}^{-1}$ vibronic progression, respectively. This result has been connected by Ernsting et al. [2] to mode coupling or Duschinsky rotation. Recently, Eom and Joo [19] reported theoretical normal-modeprojected displacements of C153 25 vibrational modes of frequencies from $25 \mathrm{~cm}^{-1}$ to $500 \mathrm{~cm}^{-1}$. Additionally, they provided frequencies of 5 modes visible in the initial $900 \mathrm{fs}$ of the fluorescence decay of $\mathrm{C} 153$ in three solvents. Among all these modes, several ones corresponding to $132 \mathrm{~cm}^{-1}$ can be found. However, they are not the most active ones. Some, including the low-frequency modes $\left(27 \mathrm{~cm}^{-1}\right.$, $36 \mathrm{~cm}^{-1}$ ) have been shown to have displacements significantly higher than the modes that can be assumed to correspond to the two most active modes given by Ernsting et al. [2] of lowest frequency. It is not clear, whether these low-frequency modes take part in the molecule deactivation, however one can see that the data reported by Ernsting et al. [2], measured at a resolution of $12 \mathrm{~cm}^{-1}$, could hide a much more complicated picture of $\mathrm{C} 153$ deactivation, not attainable with the use of current fluorescence time- or frequency-resolving setups.

This paper shows that non-trivial vibrational mode coupling between $S_{1}$ and $S_{0} \mathrm{C} 153$ vibrational states can influence steady-state fluorescence and decay kinetics in solution at room temperature and below. These results are important as the unusual deactivation of $\mathrm{C} 153$ can be erroneously interpreted as an effect of intermolecular interactions. It is the change in temperature that reveals mode coupling, thus especially when used in temperature dependent studies, $\mathrm{C} 153$ deactivation should be considered as much more complicated than hitherto assumed.

Steady-state and time-resolved results for C153 dissolved in 1-chloropropane are reported. This system has been already studied by us earlier in Ref. [3], in which it has been shown that the influence of temperature on the emission spectrum position of $\mathrm{C} 153$ in five 1-chloroalkanes can be successfully modeled by the McRae continuum solvation model [20]. Ref. [3] presented exclusively the 
temperature dependence of steady-state C153 emission spectrum shape and position, examining only the intermolecular solute-solvent interactions. In this work we would like to focus on the influence of temperature on the intramolecular deactivation of $\mathrm{C} 153$, and in consequence on the temperature dependence of C153 fluorescence kinetics.

In Ref. [3] a significant change in the shape of the emission spectra with decreasing temperature was reported. This change was assumed to be related to the inhomogeneous broadening of the spectra. Here, on the basis of literature data on $\mathrm{C} 153$ vibrational states, the source of the change in shape has been clarified and evaluated. The data obtained from vibronic analysis of the emission spectra allowed an interesting analysis of the non-radiative rate $\left(k_{\mathrm{NR}}\right)$ of the probe, performed together with experimental time-resolved kinetic results. Fluorescence decays were collected at different temperatures and together with the absorption and emission spectra they were used for determination of the dependence of the fluorescence decay time $\left(\tau_{\mathrm{F}}\right)$, quantum yield $\left(\varphi_{\mathrm{F}}\right)$, radiative $\left(k_{\mathrm{F}}\right)$ and nonradiative rates of $\mathrm{C} 153$ in $\mathrm{ClP}$. All these results have thrown new light on the temperature dependence of deactivation of $\mathrm{C} 153$ in ClP, and probably also in other weakly interacting solvents. The results presented are important for several reasons: a) they prove the complexity of character of $\mathrm{C} 153$ deactivation process, as shown earlier by Ernsting et al. [2] using different experimental methods, b) they show the usefulness of temperature dependent studies of well known dyes, assumed to be almost completely characterized, c) they indicate how much information about the vibrational deactivation can be determined from steady-state absorption/emission spectra in a broad range of temperature in a solvent environment.

\section{Materials and Methods}

The chemicals used in the study were the same as in the previous paper [3]. C153 (Fluka) was used as received. 1chloropropane 99\% (ClP, Aldrich), was dehydrated before use over the $3 \AA$ (Merck) and $4 \AA$ (Fluka) molecular sieves. The sample was prepared in argon atmosphere. C153 concentration was kept at the level of $10^{-6}-10^{-5} \mathrm{M}$.

All measurements were carried using an Oxford Instruments OptistatDN cryostat. Absorption spectra were measured using a Jasco V-550 spectrophotometer adapted to be used with the OptistatDN cryostat. Emission spectra were measured on a home-modernized Aminco SPF-500 spectrofluorimeter with a single photon counting system. The excitation wavelength was set to the maximum of the $S_{0} \rightarrow S_{1}$ absorption band at a selected temperature. Fluorescence quantum yield at room temperature was determined by a relative method using quinine sulfate in $0.1 \mathrm{~N} \mathrm{H}_{2} \mathrm{SO}_{4}$ $\left(\varphi_{\mathrm{F}}=0.52\right)$ as a standard. At other temperatures, using the same $\mathrm{C} 153$ in CIP solution, quantum yields were determined by using the $\varphi_{\mathrm{F}}$ values found at room temperature as reference. Fluorescence decays were collected using a timecorrelated single photon counting system (TCSPC) [21]. The excitation wavelength was set to the maximum of the $S_{0} \rightarrow S_{1}$ absorption band at room temperature. This approach was chosen to avoid inevitable changes in excitation pulse shape and in consequence in the instrument response function (IRF), that accompany the changes in the excitation wavelength in our setup. Potential implications of such a decision are discussed at the end of the paper. The decays were collected in 2000 channels. The time per channel was set to 12.2 ps and the scattered signal of Ludox in water solution was used as the IRF of the TCSPC system. The full width at half of the IRF maximum (fwhm) was $\sim 30$ ps (measured with a 0.61 ps per channel time resolution). The simplex optimization routine was used in the decay fitting procedure. The decays were collected until 10,000 counts were obtained in the maximum, while the IRF was collected up to 40,000 counts in the maximum.

\section{Calculations}

\section{Steady-state Spectra}

According to the Kennard-Stepanov relation [22-24], the emission spectrum position and shape can be connected to the absorption spectrum position and shape in the way described by Knox and Sawicki [25]. In short, using the Einstein model of density of the field modes and the relation between the $\mathrm{A}$ and $\mathrm{B}$ Einstein coefficients, we can define a function of frequency, depending on the absorption and emission spectra of a probe in a homogeneous environment (in MKS [26]):

$F(v)=\ln \left(\frac{c^{2}}{8 \pi h} \frac{P(v)}{v^{3} \sigma(v)}\right)=-\frac{h v}{k_{\mathrm{B}} T}+\frac{h v_{0}}{k_{\mathrm{B}} T}+\ln \left(\frac{Z}{Z^{\prime}}\right)$.

$P(\nu)$ is the emissive power at a selected frequency $\nu$, $\sigma(\nu)$ is the absorption cross section at $\nu, h \nu_{0}$ is the energy gap between the lowest set of vibrational levels of the electronic states of the probe, $S_{0}$ and $S_{1}, Z$ and $Z$ ' are the partition functions for $S_{0}$ and $S_{1}$. The right side of Eq. 1 can be written as $-\frac{h v}{k_{\mathrm{B}} T}+D(T)$. By taking the derivative of $F$ $(\nu)$ with respect to $\nu$ one obtains the formula for the Kennard-Stepanov spectral temperature $\mathrm{T}^{*}$ :

$\mathrm{T}^{*}(v)=-\left[\frac{k_{\mathrm{B}}}{h} \frac{d}{d v} \ln \left(\frac{c^{2}}{8 \pi h} \frac{P(v)}{v^{3} \sigma(v)}\right)\right]^{-1}$. 
KS relation predicts a constant $T^{*}(\nu)$ dependence which up to date, as reported in Ref. [25], has not been found for any experimental system studied. However, $T^{*}(\nu)$ can give some indications on the number of absorbing/emitting species (manifolds) and the vibronic distribution and relaxation in $S_{1}$. The presence of peaks in $\mathrm{T}^{*}(\nu)$ suggest the presence of more then one manifold, whose number can be related to the number of the peaks. Difference between $\mathrm{T}^{*}(\nu)$ and the measured $\mathrm{T}$ (usually $\mathrm{T}^{*}(\nu)$ is higher) indicates emission from vibrationally non-relaxed states. Thus, this model has been used to make a preliminary test of the nature of $\mathrm{C} 153$ relaxation.

The emission spectra have been also analyzed using the model described in Ref. [27]. On the basis of this model, the emission spectrum of the molecule in a homogeneous non-interacting solvent can be defined as (MKS):

$$
I(v)=\sum_{n_{1}} \sum_{n_{2}} \ldots \sum_{n_{\mathrm{N}}} I_{n_{1} n_{2} \ldots n_{\mathrm{N}}}(v)
$$

$$
\begin{aligned}
& I_{n_{1} n_{2} \ldots n_{\mathrm{N}}}(v)=\left(\frac{E_{00}-\sum_{\mathrm{j}=1}^{\mathrm{N}} n_{\mathrm{j}} h \bar{v}_{\mathrm{j}}}{E_{00}}\right)^{3} \prod_{\mathrm{j}=1}^{\mathrm{N}}\left(\frac{\xi_{\mathrm{j}}^{n_{\mathrm{j}}}}{n_{\mathrm{j}} !}\right) \\
& \exp \left[-4(\ln (2))\left(\frac{h v-E_{00}+\sum_{\mathrm{j}=1}^{\mathrm{N}} n_{\mathrm{j}} h \bar{v}_{\mathrm{j}}}{\Delta \bar{v}_{1 / 2}}\right)^{2}\right]
\end{aligned}
$$

$\xi_{\mathrm{j}}=1 / 2\left(\Delta_{\mathrm{j}}\right)^{2}$

where $\Delta_{j}$ is the change in bond equilibrium distance between the excited and ground states. Note that the third power in Eq. 4 in place of the fourth, as used in Ref. [27], indicates that the emission intensity is given in units of the number of quanta emitted per energy interval [28]. In theory, the model uses all optically active vibrational modes present in the probe molecule. Obviously, the number of modes is truncated to $\mathrm{N}$ most active accepting vibrational modes in the ground state. Similarly to $h \nu_{0}$ in Eq. $1, E_{00}$ is the energy gap between the lowest levels of the fitted vibronic modes of $S_{0}$ and $S_{1}$ states. The parameter $n_{\mathrm{j}}$ is the vibronic number of the $\mathrm{j}$-th accepting mode. The summations in Eq. 3 has been limited to small values, high enough to properly model the spectra. The symbol $\bar{v}_{\mathrm{j}}$ is the $\mathrm{j}$ accepting mode frequency, $\nu$ is the frequency at which the emission spectrum is measured, $\Delta \bar{v}_{1 / 2}$ is the full width at half-maximum of the Gaussian inhomogeneous broadening, and $\xi_{\mathrm{j}}$ is the Huang-Rhys vibrational coupling factor related to the equilibrium bond distance difference between the excited and ground states. Depending on the selected strategy one can try to determine $I_{n_{1} n_{2} \ldots n_{\mathrm{j}}}(v)$ from the quantum mechanically calculated and/or experimentally determined parameter values. The function given in Eq. 3 can also serve as a fitting model. The last approach was chosen in this paper. Several different fitting procedures were tested. Up to three vibrational modes were used. Their frequencies were fitted or fixed to literature values reported by Topp et al. [1], and later by Ernsting et al. [2], given in the experimental section together with the results of the fit. The maximum vibronic number $\mathrm{N}$ was varied from 1 to 10 . $E_{00}, \xi_{\mathrm{j}}, \Delta \bar{v}_{1 / 2}$ and a scaling $a_{0}$ factor (by which $I(\nu)$ was multiplied) were always treated as parameters of the fit. The quality of the fit was estimated using the $\chi^{2}$ value and the errors of the fitted parameters values.

\section{Kinetic Data}

Absorption and emission spectra along with fluorescence decay times and quantum yield values were used for determination of the absorption $|M|_{\mathrm{g} \rightarrow \mathrm{e}}^{2}$ and emission $|M|_{\mathrm{e} \rightarrow \mathrm{g}}^{2}$ transition dipole moments modulus squared (CGS) [29].

$|M|_{\mathrm{g} \rightarrow \mathrm{e}}^{2}=\frac{1}{n} \frac{2303}{8 \pi^{3}} \frac{3 h c}{N_{A}} \int \frac{\zeta(v)}{v} d v$

$|M|_{\mathrm{e} \rightarrow \mathrm{g}}^{2}=\frac{3 h}{64 \pi^{4}} \frac{1}{n^{3}} k_{F} \widetilde{v}_{F}^{-3}$

$\widetilde{v}_{\mathrm{F}}^{-3}=\frac{\int I(v) v^{-3} d v}{\int I(v) d v}$

$k_{\mathrm{F}}=\frac{\varphi_{\mathrm{F}}}{\tau_{\mathrm{F}}}$.

Above, $\zeta(\nu)$ is the molar extinction coefficient, $k_{\mathrm{F}}$ is the radiative rate constant and $I(\nu)$ is the fluorescence intensity at frequency $\nu$. Time-resolved fluorescence decays were used to determine transition dipole moments but first of all to compare the experimentally determined non-radiative rates with those derived from the Kober et al. model [28]. The rates found experimentally were determined in an ordinary way by using the fluorescence quantum yields and decay times:

$k_{\mathrm{NR}}=\frac{1}{\tau_{\mathrm{F}}}-k_{\mathrm{F}}$

The model developed in Ref. [28] is a simplified version of the model of the non-radiative deactivation provided by 
Jortner, Freed and Englman [30, 31]. In Ref. [30, 31], a constant frequency of the same vibrational modes in the ground and excited electronic state is assumed. The deactivation takes place by transition from the $S_{1}{ }^{(\mathrm{k})}$ state to the $S_{0}{ }^{(\mathrm{k})}$ state, which corresponds to a coupling of a $\nu_{\mathrm{k}}$ promoting mode in the excited and ground electronic states. Next, the excess energy in $S_{0}$ is distributed among the $\nu_{\mathrm{j}}$ accepting modes. Assuming the presence of only one, low frequency promoting mode, and one active accepting mode, $\bar{v}_{\mathrm{A}}$, the non-radiative decay rate in a homogeneous noninteracting solvent can be defined as (Ref. [28], CGS):

$$
\begin{aligned}
k_{\mathrm{NR}}= & C_{\mathrm{k}}^{2} v_{\mathrm{k}} \sqrt{\frac{\pi}{2 \bar{v}_{\mathrm{A}} E_{00}}} \exp \left(-\xi_{\mathrm{A}}\right) \\
& \times \exp \left(-\frac{\gamma E_{00}}{v_{\mathrm{A}}}+(\gamma+1)^{2}\left(\frac{\Delta \bar{v}_{1 / 2}}{\bar{v}_{\mathrm{A}}}\right)^{2} /(16 \ln (2))\right)
\end{aligned}
$$

$\gamma=\ln \left(\frac{E_{00}}{\xi_{\mathrm{A}} \bar{v}_{\mathrm{A}}}\right)-1$

$C_{\mathrm{k}}$ is the electronic coupling between the electronic states participating in the transition through the promoting mode and other parameters are defined as in Eq. 4 (with index $j=A$ ). The simplifying assumptions used when developing Eq. 11 were as follows: (1) presence of one effective promoting mode, $\nu_{\mathrm{k}}$; (2) no thermal population of the promoting and accepting modes in either the ground or excited state. Clearly, assumption (2) may not be applicable to the data presented in this paper. However, as will be shown, the model gives reasonable qualitative results. As $C_{\mathrm{k}}$ and $\nu_{\mathrm{k}}$ are unknown, they have been set to 1 , thus the calculations could only be used to check the temperature influence on the rest of Eq. 12. Thus, no direct theoretical (in fact semi-empirical) values of $k_{\mathrm{NR}}$ could be reported, but a comparison with the experimental $k_{\mathrm{NR}}(\mathrm{T})$ dependence allowed some conclusions on the sources of changes in $\tau_{\mathrm{F}}$ and $\varphi_{\mathrm{F}}$ with temperature as presented in the next section.

\section{Experimental Results}

Figure 1 presents $\mathrm{C} 153$ absorption $S_{0} \rightarrow S_{1}$ band in 1chloropropane at different temperatures from the range 183-323 K. Decreasing temperature makes the oscillator strength of the transition mentioned increase. A slight change in the shape and position of the spectrum, bathochromic in character, is also observed.

Obviously, absorption spectra were truncated at a wavelength corresponding to the minimum signal between the $S_{0} \rightarrow S_{1}$ band and the next blue one. Figure 2 presents

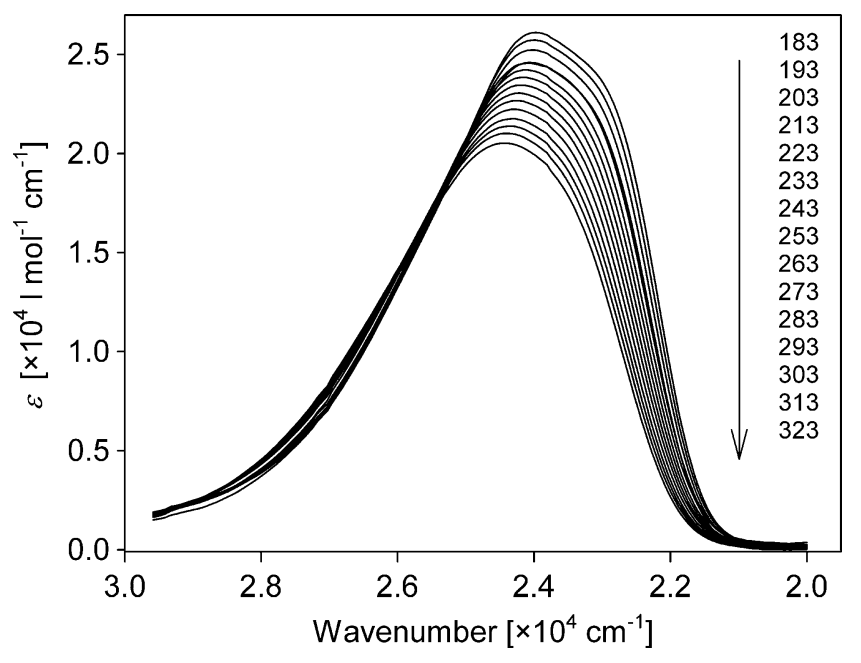

Fig. $1 \mathrm{C} 153$ in $\mathrm{ClP}$ absorption spectra

the $\mathrm{KS} \mathrm{T}^{*}(\nu)$ dependencies. $\mathrm{T}^{*}(\nu)$ was calculated numerically with a derivative window of 10 points. As reported by Sawicki and Knox [25], it has been found that smaller windows lead to the same $\mathrm{T}^{*}(\nu)$ curve shape but the noise increases with the narrowing window width.

Extending the numerical derivation step (Eq. 2) above 10 did not change $\mathrm{T}^{*}(\nu)$ significantly. As can be seen in Fig. 2, in some range of frequencies $\mathrm{T}^{*}(\nu)$ has values equal or slightly smaller than the actual temperature. To check the influence of the noise in $F(\nu)$ on $\mathrm{T}^{*}(\nu)$, the 4th-order polynomial curves were fitted to $F(\nu)$ and their analytical derivatives were used in $\mathrm{T}^{*}(\nu)$ determination. Such $\mathrm{T}^{*}(\nu)$ "noiseless" curves were found very similar to those shown in Fig. 2. $T^{*}(\nu)$ dependence shows no peaks or singularities. $\mathrm{T}^{*}(\nu)$ values seem smaller from the actual temperature by $\sim 10 \mathrm{~K}$. The values found at the lowest temperature reach

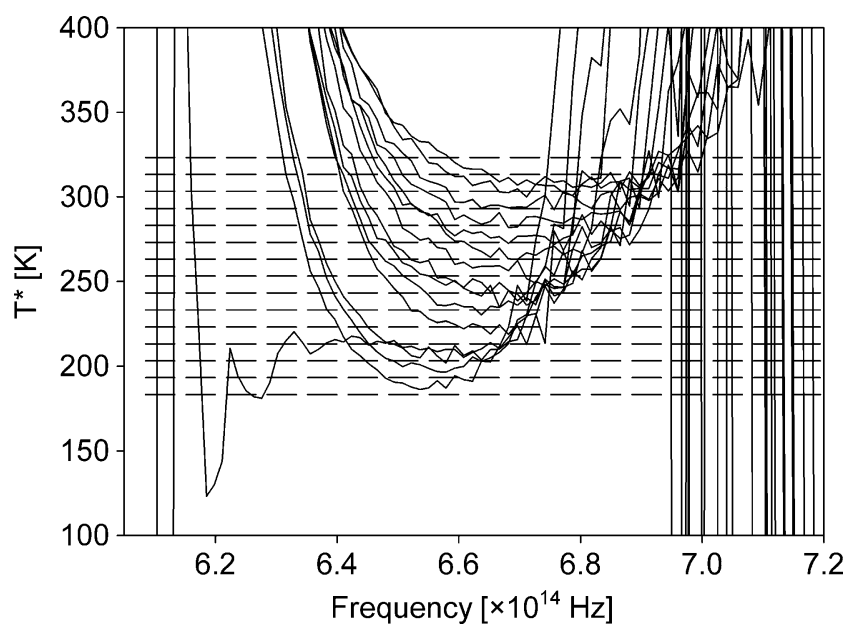

Fig. 2 Kennard-Stepanov $\mathrm{T}^{*}(\nu)$ dependence zoomed to the region of interest (dash lines correspond to subsequent temperatures at which $\mathrm{T}^{*}(\nu)$ was measured). Statistical error was estimated to be $\sim 10 \mathrm{~K}$ 
their laboratory counterpart but it is not clear if it is not related to the narrowing of the range in which $\mathrm{T}^{*}(\nu)$ values are comparable to the actual temperature. Here, it is hard to conclude whether the inequality $\mathrm{T}^{*}(\nu)<\mathrm{T}$ is significant enough to deserve interpretation. The results lead however to an important conclusion that a thermal equilibration of the $S_{1} \mathrm{C} 153$ state in CIP is reached before fluorescence takes place at each temperature, and no indication of multiple emitting states can be found in the $\mathrm{T}^{*}(\nu)$ dependence, in accordance to Ref. [5] results.

At first glance, similar standard picture of the $\mathrm{C} 153$ emission process has been found as a result of the fit of the function given in Eq. 3 to the emission spectra. Figure 3 presents the spectra and the curves fitted to them at three selected temperatures.

It has been found that the most reliable results, concluded as such on the basis of $\chi^{2}$ values and errors of the fitted parameters' values, were obtained when using only one accepting vibrational mode, $\bar{v}_{\mathrm{A}}$. It held true irrespective of whether the mode frequency was fitted or fixed during the fit. A vibrational progression of $N=5$ was found to be the most suited to recreate the experimental data. The frequency of the active vibrational mode was found to be in the range $1,006-1,174 \mathrm{~cm}^{-1}$. This range corresponds well to the $1,150 \mathrm{~cm}^{-1}$ frequency of the mode reported by Ernsting et al. [2] as the most intense in the fluorescence oscillator distribution of jet-cooled C153. Also two next most active vibrational modes, reported by these authors, $810 \mathrm{~cm}^{-1}$ and $360 \mathrm{~cm}^{-1}$, were used additionally during the fit, together with the $1,150 \mathrm{~cm}^{-1}$ mode. However, their presence has been found to blur the result of the fit, as it led to an increase in the errors of the parameters' values. Thus $E_{00}, \bar{v}_{\mathrm{A}}, \Delta \bar{v}_{1 / 2}, \xi_{\mathrm{A}}$ and $a_{0}$ were the parameters of the fit. Table 1 gives the values of these

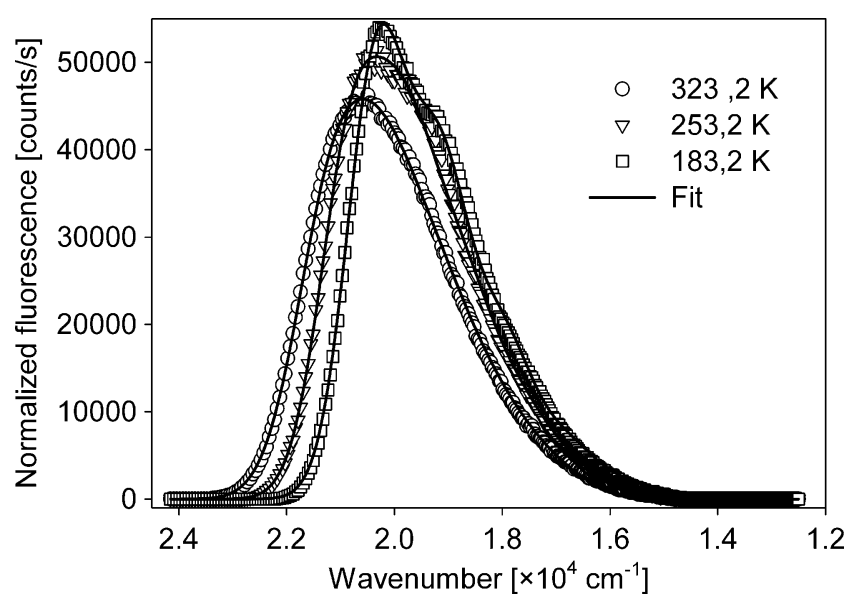

Fig. 3 Experimental emission spectra of $\mathrm{C} 153$ in $\mathrm{ClP}$ at selected temperatures (323.2 K circles, $253.2 \mathrm{~K}$ triangles, $183.2 \mathrm{~K}$ squares) and fitted curves of the function given in Eq. 3 (solid line) parameters at subsequent temperatures set during the experiment.

The values of all parameters correspond quite properly to what should be expected from analysis of Topp et al. [1] and Ernsting et al. [2] works. The latter authors report the three modes mentioned above, $1,150 \mathrm{~cm}^{-1}, 360 \mathrm{~cm}^{-1}$ and $810 \mathrm{~cm}^{-1}$, as the most active together with the fourth mode at $132 \mathrm{~cm}^{-1}$. This last mode has been omitted by them in the modeling of the $\mathrm{C} 153$ emission spectra, as the mode subsumed into the broadening function of the $1,150 \mathrm{~cm}^{-1}$ mode. The results of the fit reported above indicate that the $1,150 \mathrm{~cm}^{-1}$ mode broadening hides also the two other most active vibrational modes in CIP solution, $360 \mathrm{~cm}^{-1}$ and $810 \mathrm{~cm}^{-1}$. The displacement of the $1,150 \mathrm{~cm}^{-1}$ mode given in Ref. [2] corresponding to $\xi_{\mathrm{A}}$ is slightly smaller than the one given in Table 1. This fact can be related to the difference in the model used in emission simulation, but primarily it is simply connected to the fact that Ernsting et al. studied cooled jet spectra with much better resolved vibrational distribution, in gas phase. A comparison of the results of the fit with $\bar{v}_{\mathrm{A}}$ fixed to $1,150 \mathrm{~cm}^{-1}$ with those given in Table 1 have shown $\xi_{\mathrm{A}}$ to be correlated with $\Delta \bar{v}_{1 / 2}$. When $\bar{v}_{\mathrm{A}}$ is fixed, the only parameter not showing monotonic change with temperature is $\xi_{\mathrm{A}}$, while it is not the case for the results presented in Table 1 . Here $\bar{v}_{\mathrm{A}}$ and $\xi_{\mathrm{A}}$ have a common extreme at $293 \mathrm{~K}$, as shown in Fig. 4.

Later it is shown that this effect has physical source and is not simply a result of correlations between fitted parameters. Generally, temperature changes of all parameters given in Table 1 follow the expected way. Due to decreasing energy of the solute-solvent interactions, $E_{00}$ rises with temperature. As observed by Ernsting et al., $\xi_{\mathrm{A}}$ and $\Delta \bar{v}_{1 / 2}$ follow the same temperature dependence as $E_{00}$. The values of $a_{0}$ fall with temperature, which is connected primarily with the drop in absorbance with temperature rising, as shown in Fig. 1. The value of $\Delta \bar{v}_{1 / 2}$ is higher than the values given in Ref. [2], but it is a consequence of a stronger broadening related to solute-solvent interactions taking place in ClP and absent in the gas-phase.

Estimation of the electronic coupling between two singlet states can be made by determining the absorption and emission transition moments, given in Eqs. 6 and 7. To compute $|M|_{\mathrm{e} \rightarrow \mathrm{g}}^{2}$ the value of $k_{\mathrm{F}}$ was needed (Eq. 9). Thus, the fluorescence decay time, $\tau_{\mathrm{F}}$, and fluorescence quantum yield, $\varphi_{\mathrm{F}}$, were determined. Figure 5 shows temperature dependencies of both quantities.

As can be seen, the values of $\tau_{\mathrm{F}}$ and $\varphi_{\mathrm{F}}$ cannot be interpreted in the same simple way as the data reported earlier in this work. It must be stressed that the values of both parameters were determined using different setups, $\varphi_{\mathrm{F}}$ with Jasco V-550 and Aminco SPF-500 spectrometers, while $\tau_{\mathrm{F}}$ with the TCSPC system. The only common part was the cryostat and the object of study. The sample was 
Table $1 E_{00}, \bar{v}_{\mathrm{A}}, \Delta \bar{v}_{1 / 2}, \xi_{\mathrm{A}}$ and $a_{0}$ values obtained as a result of the fit of the function given in Eq. 3 to the emission spectra of $\mathrm{C} 153$ in ClP measured at selected temperatures

\begin{tabular}{|c|c|c|c|c|c|c|}
\hline \multirow{15}{*}{$\begin{array}{l}\text { Table } 1 E_{00}, \bar{v}_{\mathrm{A}}, \Delta \bar{v}_{1 / 2}, \xi_{\mathrm{A}} \text { and } \\
a_{0} \text { values obtained as a result } \\
\text { of the fit of the function given in } \\
\text { Eq. } 3 \text { to the emission spectra } \\
\text { of } \mathrm{C} 153 \text { in } \mathrm{ClP} \text { measured at } \\
\text { selected temperatures }\end{array}$} & $\mathrm{T}[\mathrm{K}]$ & $E_{00}\left[\mathrm{~cm}^{-1}\right]$ & $\Delta \bar{v}_{1 / 2}\left[\mathrm{~cm}^{-1}\right]$ & $\bar{v}_{\mathrm{A}}\left[\mathrm{cm}^{-1}\right]$ & $\xi_{\mathrm{A}}$ & $a_{0}$ \\
\hline & 323 & $22,210 \pm 50$ & $1,570 \pm 10$ & $1,030 \pm 10$ & $2,14 \pm 0,02$ & $16,850 \pm 300$ \\
\hline & 313 & 22,170 & 1,535 & 1,025 & 2,13 & 17,340 \\
\hline & 303 & 22,120 & 1,495 & 1,010 & 2,17 & 17,230 \\
\hline & 293 & 22,100 & 1,445 & 1,005 & 2,24 & 16,920 \\
\hline & 283 & 22,015 & 1,440 & 1,020 & 2,15 & 19,130 \\
\hline & 273 & 21,970 & 1,410 & 1,010 & 2,16 & 19,810 \\
\hline & 263 & 21,920 & 1,390 & 1,015 & 2,15 & 19,900 \\
\hline & 253 & 21,830 & 1,385 & 1,025 & 2,10 & 21,180 \\
\hline & 243 & 21,810 & 1,360 & 1,060 & 2,03 & 23,050 \\
\hline & 233 & 21,730 & 1,345 & 1,045 & 2,02 & 23,450 \\
\hline & 223 & 21,710 & 1,340 & 1,090 & 1,94 & 25,920 \\
\hline & 213 & 21,640 & 1,320 & 1,070 & 1,93 & 26,070 \\
\hline & 203 & 21,630 & 1,310 & 1,125 & 1,83 & 29,630 \\
\hline & 193 & 21,570 & 1,300 & 1,165 & 1,76 & 33,530 \\
\hline $\begin{array}{l}\text { a A decrease of the error at low } \\
\text { temperatures was observed }\end{array}$ & 183 & 21,500 & 1,290 & 1,175 & $1,72 \pm 0,01^{\mathrm{a}}$ & 34,360 \\
\hline
\end{tabular}

${ }^{\mathrm{a}} \mathrm{A}$ decrease of the error at low temperatures was observed

prepared separately for determination of $\tau_{\mathrm{F}}(\mathrm{T})$ and $\varphi_{\mathrm{F}}(\mathrm{T})$. We can exclude erroneous operation of the cryostat, as measurements of $\tau_{\mathrm{F}}(\mathrm{T})$ in hexafluoroisopropanol and trifluoroethanol showed a simple monotonic change in $\tau_{\mathrm{F}}$ with temperature. On the other hand, in propionitrile the values of $\tau_{\mathrm{F}}$ similar to those shown in Fig. 5 were observed. These results will be presented elsewhere, here it should be noted that the two protic solvents can form strong hydrogen bonds while propionitrile cannot, therefore such differences in $\tau_{\mathrm{F}}(\mathrm{T})$ are possible. Discontinuous changes in temperature dependences are typical of phase-transition phenomena. It is most probable that a phase-transition in CIP would lead to visible changes in the absorption and especially in the emission spectra of C153. For instance, such observation was made by the author for 4-aminopthalimide dissolved in 1-chloroalkanes not properly dehydrated. As no such changes were observed it was assumed that the surprising

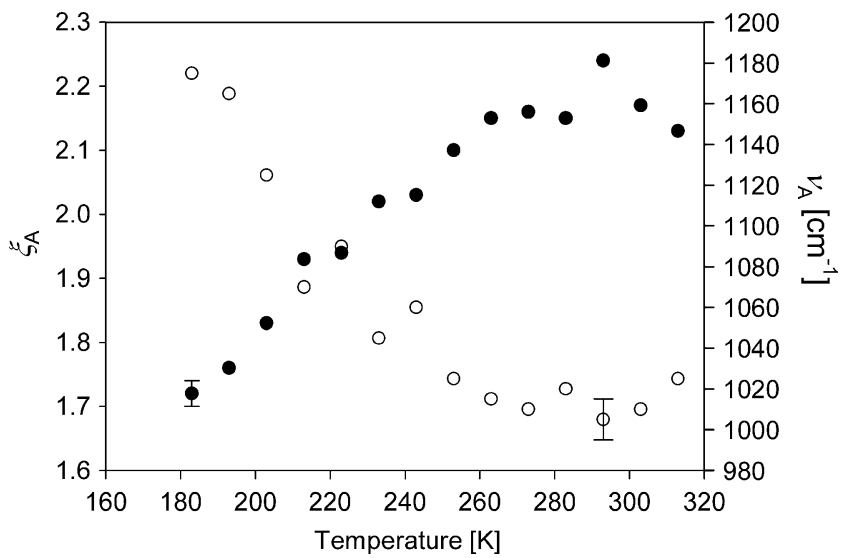

Fig. 4 Temperature dependence of the accepting mode displacement $\left(\xi_{\mathrm{A}}\right.$, filled circles $)$ and frequency $\left(\bar{v}_{\mathrm{A}}\right.$, empty circles $)$ as obtained from fit of $\mathrm{C} 153$ emission spectra to the model given in Eqs. 3-5
$\tau_{\mathrm{F}}(\mathrm{T})$ and $\varphi_{\mathrm{F}}(\mathrm{T})$ dependencies must be related to some intra-molecular changes in the deactivation process controlled by temperature. However, the abrupt change in the character of the dependence is most probably only related to the small temperature resolution of the experiment. These results lead to $k_{\mathrm{F}}$ and $k_{\mathrm{NR}}$ (Eq. 10) values presented in Fig. $6 ; k_{\mathrm{F}}$ is approximately three times higher than $k_{\mathrm{NR}}$. There are two temperatures at which drastic changes in $k_{\mathrm{F}}(\mathrm{T})$ and $k_{\mathrm{NR}}(\mathrm{T})$ take place: $270 \mathrm{~K}$ and $300 \mathrm{~K}$. These changes take place in the temperature range at which only the changes in $\xi_{\mathrm{A}}(\mathrm{T})$ and $\bar{v}_{\mathrm{A}}(\mathrm{T})$ are observed (Fig. 4). The ratio of the minimal vs maximal $k_{\mathrm{F}}$ equals only 0.97 , while for $k_{\mathrm{NR}}$ this ratio is 0.75 . However, because of higher values of $k_{\mathrm{F}}$ the absolute change in $k_{\mathrm{NR}}$ is only about twice as big as that in $k_{\mathrm{F}}$.

With $k_{\mathrm{F}}(\mathrm{T})$ determined, the emissive transition moment, $M_{\mathrm{e} \rightarrow \mathrm{g}}$, was calculated along with the absorption one, $M_{\mathrm{g} \rightarrow \mathrm{e}}$.

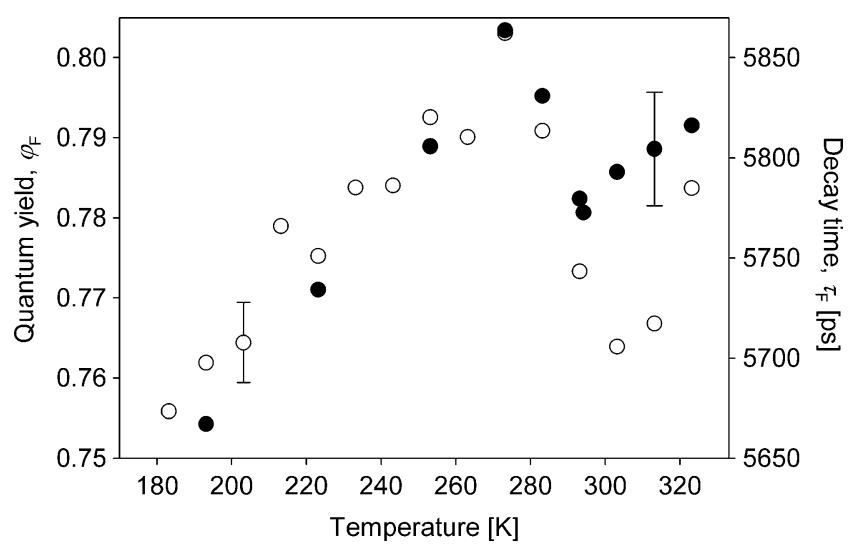

Fig. 5 Fluorescence decay time (filled circles) and quantum yield (empty circles) vs temperature. Vertical lines indicate estimated errors of the values of the corresponding parameters 


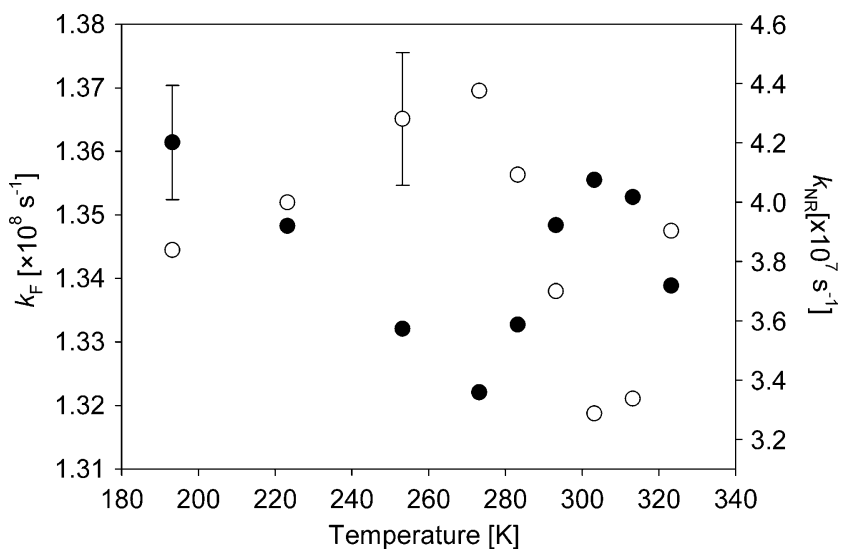

Fig. 6 Radiative (empty circles) and non-radiative (filled circles) rates vs temperature. Vertical lines indicate estimated errors of the corresponding values of the parameters

Figure 7 presents the temperature dependencies of these quantities modulus squared, indicating the probability of absorption and emission as functions of temperature.

The values are similar to the ones reported in Ref. [5]. In accordance to what could be concluded from the changes in signal intensity in the absorption spectra (Fig. 1), reducing temperature leads to a rise in the absorption probability. The dependence is monotonic (disregarding one clearly erroneous point). Thus, the $M_{\mathrm{g} \rightarrow \mathrm{e}}(\mathrm{T})$ dependence is quite ordinary and can be connected to a drop in the $\mathrm{S}_{0} \rightarrow \mathrm{S}_{1}$ energy gap with decreasing temperature. The $M_{\mathrm{e} \rightarrow \mathrm{g}}(\mathrm{T})$ dependence follows the dependence of $k_{\mathrm{F}}(\mathrm{T})$. Nevertheless, the change in $M_{\mathrm{g} \rightarrow \mathrm{e}}$ is an order of magnitude larger than that in $M_{\mathrm{e} \rightarrow \mathrm{g}}$. Now, an attempt to model the temperature dependence of $k_{\mathrm{NR}}$ was made. Figure 8 presents $k_{\mathrm{NR}}(\mathrm{T})$ obtained with Eq. 11, assuming $C_{\mathrm{k}}=\nu_{\mathrm{k}}=1$, and once again experimental $k_{\mathrm{NR}}(\mathrm{T})$ is shown, for the sake of comparison. Arbitrary units are used in the figure to underline the relativity of the $k_{\mathrm{NR}}$ theoretical values.

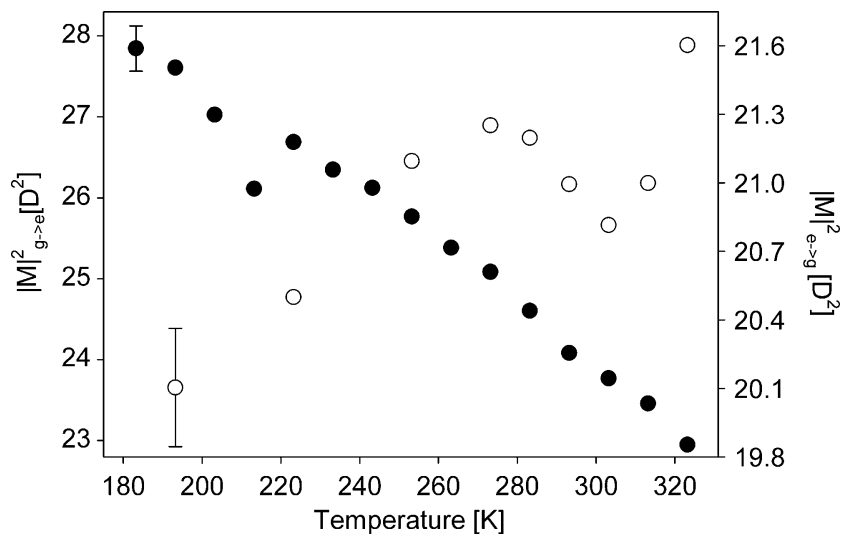

Fig. 7 Absorption (filled circles) and emission (empty circles) modulus squared of the transition dipole moments. Vertical lines indicate estimated errors of the values of the corresponding parameters

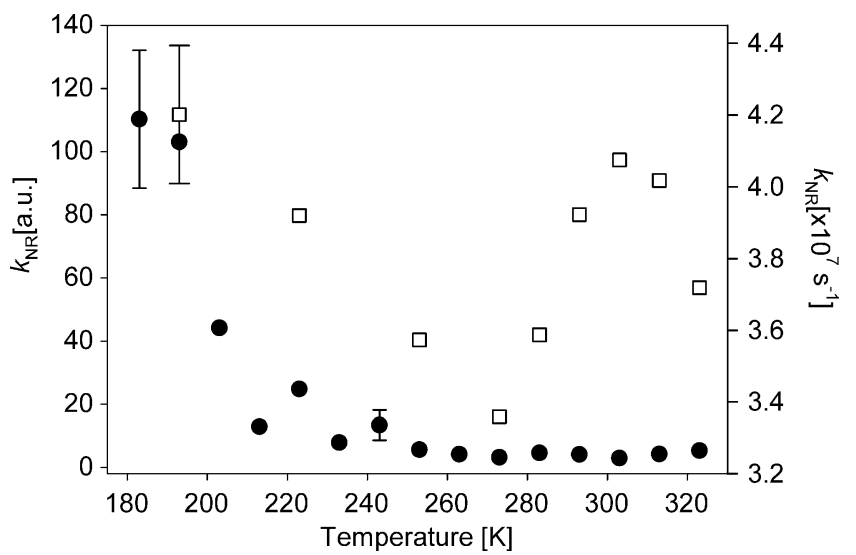

Fig. 8 Non-radiative deactivation rate for $\mathrm{C} 153$ in $\mathrm{ClP}$ at different temperatures determined using Eq. 11 (filled circles, left axis) and Eq. 10 (empty squares, right axis). Electronic coupling term, $C_{\mathrm{K}}$, and promoting mode, $\nu_{\mathrm{k}}$, are assumed to be equal 1. Estimated errors of the values were found to be of the order of the values, see details in the text

At $\mathrm{T} \leq 273 \mathrm{~K}, k_{\mathrm{NR}}$ determined in both ways falls with rising temperature. As far as the theoretical $k_{\mathrm{NR}}$ is concerned, the temperature dependence corresponds well to what could be expected from the non-radiative deactivation energy-gap law. To evaluate the $C_{\mathrm{k}}{ }^{2} \cdot \nu_{\mathrm{k}}$ term of Eq. 11 , assumed until now to be equal 1 , the experimental $k_{\mathrm{NR}}$ (Eq. 10) has been divided by the theoretical one (Eq. 11). Figure 9 presents this relation.

The $C_{\mathrm{k}}^{2} \cdot v_{\mathrm{k}}$ temperature dependence indicates a significant change in the coupling between promoting mode vibrational states in $S_{1}$ and $S_{0}$. It can also be related to a change in the main active promoting mode or both. Finally, one cannot exclude the erroneousness of the assumption of the presence of a single promoting mode taken to use Eq. 11 to evaluate $k_{\mathrm{NR}}$.

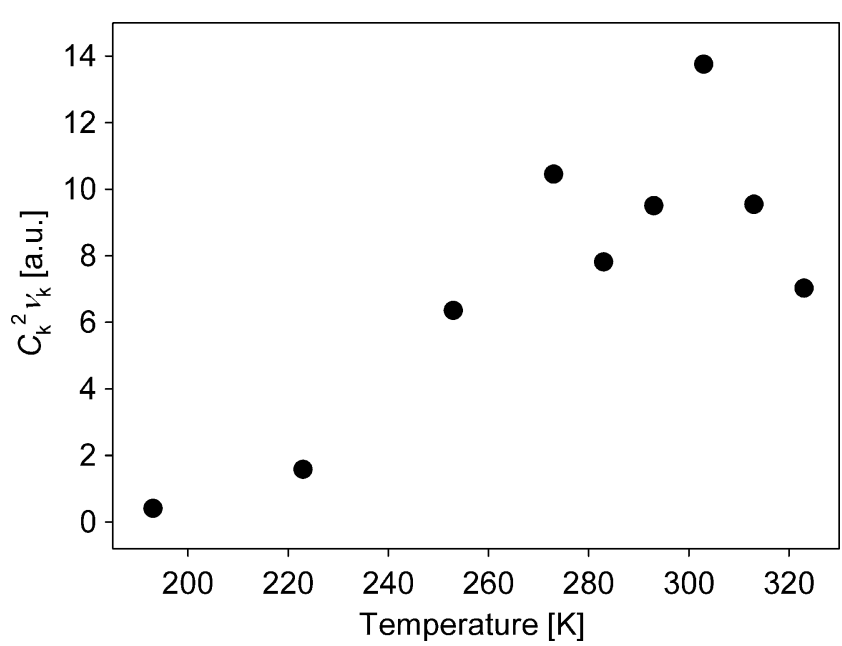

Fig. 9 Temperature dependence of the $C_{\mathrm{k}}^{2} \cdot v_{\mathrm{k}}=\frac{k_{\mathrm{NR}}^{\exp }}{k^{\text {theor }}}$ ratio, $k_{\mathrm{NR}}^{\exp }$ being the non-radiative deactivation rate determined from Eq. 10 and $k_{\text {theor }}^{\text {exp }}$ the same determined from Eq. 11 


\section{Discussion}

Changes in $k_{\mathrm{F}}$ and $M_{\mathrm{e} \rightarrow \mathrm{g}}$ with temperature clearly indicate a non-trivial change in electronic coupling between the optically active vibrational modes in $S_{1}$ and $S_{0}$. For $M_{\mathrm{g} \rightarrow \mathrm{e}}(\mathrm{T})$ it is the opposite, the dependence is classical. If assuming, as in Ref. [2], conformer I to be the species active in absorption, one could relate the $M_{\mathrm{e} \rightarrow \mathrm{g}}(\mathrm{T})$ dependence to the barrier activated conformational change in C153 from conformer I to II. However, such an interpretation is not supported by the gas-phase results [2], and should lead to a much simpler $k_{\mathrm{NR}}(\mathrm{T})$ dependence (Fig. 8). As mentioned above, the $1,150 \mathrm{~cm}^{-1}$ mode was assumed to cover, due to inhomogeneous broadening, the non-trivial $132 \mathrm{~cm}^{-1}$ mode revealed in Refs [1, 2]. For this lowfrequency mode the assumption of the absence of thermal occupation in $S_{1}$, taken when using Eqs. 3 and 11, is certainly wrong. As Ernsting et al. [2] found the optical activity of this mode be strongly dependent on the excitation light energy, it can be concluded that its temperature dependent occupation may lead to some untypical results like the one reported in this paper. As mentioned in the introduction section, Eom and Joo [19] reported the low-frequency modes (at $27 \mathrm{~cm}^{-1}, 36 \mathrm{~cm}^{-1}$ ) with displacements significantly greater than that of the $132 \mathrm{~cm}^{-1}$ mode. Although, we do not know if these modes are visible, it is possible that the $1,150 \mathrm{~cm}^{-1}$ mode covers not only the $800 \mathrm{~cm}^{-1}, 360 \mathrm{~cm}^{-1}$ and $132 \mathrm{~cm}^{-1}$ ones but also some other ones, during the steady-state emission spectra fit as carried out in this work. Thus, temperature most likely affects the coupling of several modes which together give the $1,150 \mathrm{~cm}^{-1}$ response as observed above. As one of low-frequency modes is highly likely to be the promoting one, it is not surprising to see that temperature affects non-trivially not only the emission (Figs. 3, 4 and 5), but also the non-radiative deactivation process (Figs. 6, 8 and 9). It is however not possible, on the basis of the results presented in this paper, to indicate which modes are the responsible for the change in coupling with temperature as shown by the fluorescence deactivation.

It is worth noting that while it is hard to find a temperature dependence of any of the fitted parameters given in Table 1 similar to those shown for $\tau_{\mathrm{F}}$ and $\varphi_{\mathrm{F}}$ in Fig. 5, a reconstruction of the $\mathrm{C} 153$ emission spectra based on Table 1 values led to a $\varphi_{\mathrm{F}}(\mathrm{T})$ dependence very similar to the experimental one. It indicates the correctness of the emission spectra fits. The only manifestation of the nontrivial $\tau_{\mathrm{F}}(\mathrm{T})$ and $\varphi_{\mathrm{F}}(\mathrm{T})$ dependencies in the fits is the change in the character of $\bar{v}_{\mathrm{A}}(\mathrm{T})$ and $\xi_{\mathrm{A}}(\mathrm{T})$ dependencies at $\mathrm{T}>273 \mathrm{~K}$ (Fig. 4), that is in the same temperature range in which the most abrupt changes in $\tau_{\mathrm{F}}(\mathrm{T}), \varphi_{\mathrm{F}}(\mathrm{T}), k_{\mathrm{F}}(\mathrm{T})$, $k_{\mathrm{NR}}(\mathrm{T})$ and $M_{\mathrm{e} \rightarrow \mathrm{g}}(\mathrm{T})$ take place. It seems that the $\xi_{\mathrm{A}}(\mathrm{T})$ dependence does not undergo important changes in shape, because the $\bar{v}_{\mathrm{A}}$ vibrational mode fitted to the emission spectrum is an average mode incorporating the $1,150 \mathrm{~cm}^{-1}$ mode as well as the $132 \mathrm{~cm}^{-1}$ one, or even lower-frequency modes. The low-frequency modes are the one found in the supersonic-jet fluorescence studies to undergo strange coupling, while the $1,150 \mathrm{~cm}^{-1}$ mode was not found to behave non-classically. Reassuming, fits to the model given by Eqs. 3-5 have shown that the dominating vibrational mode taking part in the fluorescence can hide lowerfrequency modes responsible for a non-monotonic character of the $\tau_{\mathrm{F}}(\mathrm{T})$ and $\varphi_{\mathrm{F}}(\mathrm{T})$ dependencies. This inevitably leads also to the smooth theoretical $k_{\mathrm{NR}}(\mathrm{T})$ dependence shown in Fig. 8, which results in the $C_{\mathrm{k}}^{2} \cdot v_{\mathrm{k}}$ temperature dependence (Fig. 9).

Taking into account the scale of different rate constants and the $C_{\mathrm{k}}^{2} \cdot v_{\mathrm{k}}$ temperature dependence one can finish the analysis with the following conclusion: the non-trivial deactivation a) is mainly manifested in the radiative rate, $\left.k_{\mathrm{F}}, \mathrm{b}\right)$ is also observed in the promoting mode coupling, through which it influences the non-radiative rate, $k_{\mathrm{NR}}$, c) is not observed in the high-frequency vibrational mode coupling, d) is most probably related to temperature dependent vibronic distribution of the low-frequency modes. Unfortunately, the reason for the non-trivial coupling remains unclear, as well as the promoting mode activity.

As fluorescence decays were collected after excitation of $\mathrm{C} 153$ at the wavelength corresponding to the absorption maximum at room temperature, while the emission spectra after excitation at the wavelength set to the actual temperature absorption maximum, we have to consider possible implications of this approach on the results. Excitation wavelength was changed in the range $409 \mathrm{~nm}-$ $417 \mathrm{~nm}$. This range is not wide, similarly as the difference in absorption at a selected temperature at the wavelength corresponding to the absorption maximum and at the wavelength corresponding to the maximum at room temperature. As mentioned above, absorption measurements reflected a very simple image of C153 $S_{0} \rightarrow S_{1}$ transition. On the other hand, steady-state fluorescence spectra revealed an unusual behavior, exactly the same as the one found for the fluorescence decay time. It indicates that IVR leads to the same C153 vibrational configuration, despite little differences in excitation energy. Additionally, it is consistent with the picture drawn in Ref. [2]. Although, it can be assumed that the procedure selected during the experiment did not influence the overall effect observed.

At the end it should be reminded that in Ref. [2] the authors discussed the possibility of the presence of a proximity effect, as a result of a low lying $S_{2}$ state. Such an effect could in principle affect the coupling between vibronic modes, depending on temperature and excitation energy, which in the case of the fluorescence measurements 
was growing with decreasing temperature, due to the bathochromic shift of the absorption spectrum. However, as Ernsting et al. and later Cave and Castner [6] have shown, the $S_{2}-S_{1}$ difference is in the range $0.3 \mathrm{eV}-0.8 \mathrm{eV}$, so it seems to be too large to allow any proximity effect to occur.

\section{Conclusions}

The absorption of light by C153 in ClP has been shown to be an ordinary process, growing in probability with decreasing temperature of the solution. The absorption spectra together with the emission spectra could be related in a satisfying way through the Kennard-Stepanov relation. This analysis indicated that a single electronic species absorbs the light as well as a single species emits the light. This result is in accordance with Ernsting et al. [2] observation that conformer I is the absorbing one, while conformer II is the emitting one.

Kinetic results did not show such a simple picture of C153 deactivation behavior. The fluorescence decay time, quantum yield and transition moment were found to change abruptly twice with decreasing temperature. This observation was related mainly to significant and abrupt temperature changes in the radiative deactivation rate of electronically excited C153. Low-frequency vibrational modes, one of them reported earlier in literature as showing unordinary behavior, were proposed as responsible for the observed strange deactivation temperature dependence.

Acknowledgement This study was performed under financial support of the Polish Ministry of Science and Higher Education (project N N202 091339). Dynamic studies were carried out at the Centre for Ultrafast Laser Spectroscopy at the A. Mickiewicz University in Poznań.

Open Access This article is distributed under the terms of the Creative Commons Attribution Noncommercial License which permits any noncommercial use, distribution, and reproduction in any medium, provided the original author(s) and source are credited.

\section{References}

1. Pryor BA, Palmer PM, Chen Y, Topp MR (1999) Identification of dual conformers of Coumarin 153 under jet-cooled conditions. Chem Phys Lett 299(6):536-544

2. Mühlpfordt A, Schanz R, Ernsting NP, Farztdinov V, Grimme S (1999) Coumarin 153 in the gas phase: optical spectra and quantum chemical calculations. Phys Chem Chem Phys 1:3209-3218

3. Tomczak J, Dobek K (2009) Coumarin 153 emission thermochromism studied in non-specifically and specifically interacting solvents. J Luminescence 129(8):884-891

4. Jones G II, Jackson WR, Choi C-Y, Bergmark WR (1985) Solvent effects on emission yield and lifetime for coumarin laser dyes.
Requirements for a rotatory decay mechanism. J Phys Chem 89 (2):294-300

5. Lewis JE, Maroncelli M (1998) On the (uninteresting) dependence of the absorption and emission transition moments of coumarin 153 in solvents. Chem Phys Lett 282(2):197-203

6. Cave RJ, Castner EW Jr (2002) Time-dependent density functional theory investigation of the ground and excited states of coumarins 102, 152, 153, and 343. J Phys Chem A 106 (50):12117-12123

7. Królicki R, Jarzęba W, Mostafavi M, Lampre I (2002) Preferential solvation of coumarin 153 - the role of hydrogen bonding. J Phys Chem A 106(9):1708-1713

8. Molotsky T, Huppert D (2002) Solvation statics and dynamics of coumarin 153 in hexane-propionitrile solvent mixtures. J Phys Chem A 106(37):8525-8530

9. Maroncelli M, Fleming GR (1987) Picosecond solvation dynamics of coumarin 153: the importance of molecular aspects of solvation. J Chem Phys 86(11):6221-6239

10. Bart E, Meltsin A, Huppert D (1994) Solvation dynamics of coumarin 153 in molten salts. J Phys Chem 98(13):3295-3299

11. Chapman CF, Fee RS, Maroncelli M (1995) Measurements of the solute dependence of solvation dynamics in 1-propanol: the role of specific hydrogen-bonding interactions. J Phys Chem 99 (13):4811-4819

12. Kovalenko SA, Ruthman J, Ernsting NP (1997) Ultrafast stokes shift and excited-state transient absorption of coumarin 153 in solution. Chem Phys Lett 271(1-3):40-50

13. Gustavsson T, Cassara L, Gulbinas V, Gurzadyan G, Mialocq J-C, Pommeret S, Sorgius M, van der Meulen P (1998) Femtosecond spectroscopic study of relaxation processes of three aminosubstituted coumarin dyes in methanol and dimethyl sulfoxide. J Phys Chem A 102(23):4229-4245

14. Karmakar R, Samanta A (2002) Solvation dynamics of coumarin153 in a room-temperature ionic liquid. J Phys Chem A 106 (18):4447-4452

15. Ito N, Kajimoto O, Hara K (2002) High-pressure studies of rotational dynamics for coumarin 153 in alcohols and alkanes. J Phys Chem A 106(25):6024-6029

16. Horng ML, Gardecki JA, Papazyan A, Maroncelli M (1995) Subpicosecond measurements of polar solvation dynamics: coumarin 153 revisited. J Phys Chem 99(48):17311-17337

17. Ingrosso F, Ladanyi BM, Mennucci B, Elola MD, Tomasi J (2005) Solvation dynamics in acetonitrile: a study incorporating solute electronic response and nuclear relaxation. J Phys Chem B 109 (8):3553-3564

18. Ernsting NP, Asimov M, Schäfer FP (1982) The electronic origin of the $\pi \pi^{*}$ absorption of amino coumarins studied in a supersonically cooled free jet. Chem Phys Lett 91(3):231-235

19. Eom I, Joo T (2009) Polar solvation dynamics of coumarin 153 by ultrafast time-resolved fluorescence. J Chem Phys 131 (24):244507

20. McRae EG (1957) Theory of solvent effects on molecular electronic spectra. Frequency shifts. J Phys Chem 61(5):562572

21. Karolczak J, Komar D, Kubicki J, Wróżowa T, Dobek K, Ciesielska B, Maciejewski A (2001) The measurements of pcosecond fluorescence lifetimes with high accuracy and subpicosecond precision. Chem Phys Lett 344(1-2):154-164

22. Kennard EH (1918) On the thermodynamics of fluorescence. Phys Rev 11(1):29-38

23. Kennard EH (1926) On the interaction of radiation with matter and on fluorescent exciting power. Phys Rev 28 (4):672-683

24. Stepanov BI (1957) Универсаљное соотношение между спет рами поглощения и люминесценции сложных молекул. Dokl Akad Nauk SSSR 112:839 
25. Sawicki AD, Knox RS (1996) Universal relationship between optical emission and absorption of complex systems: an alternative approach. Phys Rev A 54(6):4837-4841

26. Depending on the unit system most frequently used in the literature presenting cited in the paper equations, CGS or MKS units are used in the paper, as marked in the text

27. Caspar JV, Westmoreland TD, Allen GH, Bradley PG, Meyer TJ, Woodruff WH (1984) Molecular and electronic structure in the metal-to-ligand charge-transfer excited states of d6 transition-metal complexes in solution. J Am Chem Soc 106 (12):3492-3500
28. Kober EM, Caspar JV, Lumpkin RS, Meyer T (1986) Application of the energy gap law to excited-state decay of osmium(II)-polypyridine complexes: calculation of relative nonradiative decay rates from emission spectral profiles. J Phys Chem 90(16):3722-3734

29. Birks JB (1970) Photophysics of aromatic molecules. WileyInterscience, New York

30. Englman R, Jortner J (1970) The energy gap law for radiationless transitions in large molecules. Mol Phys 18(2):145-164

31. Freed KF, Jortner J (1970) Multiphonon processes in the nonradiative decay of large molecules. J Chem Phys 52(12):6272-6292 